\title{
PARABOLIC GREEN FUNCTIONS IN OPEN SETS
}

\author{
NEIL A. EKLUND
}

ABSTRACT. Consider the parabolic operator $L$ defined by $L u=u_{t}-$ $\left\{a_{i j} u,_{i}+d_{j} u\right\},{ }_{j}-b_{j} u,{ }_{j}-c u$ in an open set $U$ in $E^{n} \times(0, T)$. By using the natural abstraction of the notion of a Green function, the author obtains the existence of a unique Green function for $L u=0$ on $U$.

In two preceding papers the author has generalized the notion of superparabolic functions on cylinders and considered nets of superparabolic functions. These results together with the known existence of the fundamental solution in $E^{n} \times(0, T)$ and the Green function in a cylinder $Q=\Omega \times(0, T)$ are used to obtain the Green function for any domain $U \subset E^{n} \times(0, T)$.

The function $G$ will be called a Green function if it is the minimal, nonnegative, superparabolic function which can be written as a sum of the fundamental solution of $L u=0$ and a superparabolic function. This function will be shown to coincide with the Green function $\gamma(z ; \zeta)$ for the cylindrical domain $Q$ which was obtained by Aronson [1]. For $\zeta=(\xi, \tau) \in Q$, the fundamental solution $\Gamma(z ; \zeta)$ was obtained on $(x, t) \in E^{n} \times\{[0, \tau) \cup(\tau, T]\}$. To guarantee that $\Gamma(\cdot ; \zeta)$ was in $\delta_{Q}^{\prime}$ it was necessary to extend the domain of $\Gamma$ so that $\Gamma=0$ for $t=\tau$. Whenever this function is referred to, it will be in this extended sense.

Since the results obtained here depend significantly on the author's two preceding papers, definitions, theorems, and lemmas will be numbered sequentially, following those in [2]-[4]. Also, many of the proofs are identical to those for the Laplace operator given in Helms' text [6] and will not be repeated here.

Definition 13. Let $U$ be an open subset of $E^{n} \times(0, T)$ for some $T>$ 0 . A Green,function, if it exists, for $U$ is an extended real valued function $G_{U}$ on $U \times U$ with the following properties:

Presented to the Society, January 23, 1975; received by the editors April 3, 1974. AMS (MOS) subject classifications (1970). Primary 35K10; Secondary 31B05.

Key words and phrases. Second order parabolic operator, Green functions, bounded domains. 
(1) $G_{U}(z ; \zeta)=\Gamma(z ; \zeta)+H(z ; \zeta)$ where $H(\cdot ; \zeta)$ is parabolic on $U$ for each $\zeta \in U$;

(2) $G_{U} \geq 0$;

(3) if, for $\zeta \in U, v_{\zeta}$ is a nonnegative function which is the sum of $\Gamma(\bullet ; \zeta)$ and a function in $\mathcal{S}_{U}^{\prime}$, then $v_{\zeta} \geq G_{U}(\cdot ; \zeta)$.

Theorem 17. If an open set $U$ bas a Green function $G_{U}$, then it is unique.

Proof. This is an immediate consequence of property (3).

Lemma 5. If the open set $U$ bas a Green function $G_{U}$, then, for each $\zeta \in U, \inf \left\{G_{U}(z ; \zeta) ; z \in U\right\}=0$.

Proof. This is obvious from properties (2) and (3) since for any $z \in U$, $0 \leq G_{U}(z ; \zeta) \leq \Gamma(z ; \zeta)$ and $\Gamma(z ; \zeta)=0$ for $z \prec \zeta$.

A more interesting result is contained in the following theorem. The conclusion of this theorem follows from property (3) in the case where $U$ is unbounded.

Theorem 18. Let $U \subset E^{n} \times(0, T)$ be a bounded domain with Green function $G_{U}$ and let $\zeta=(\xi, \tau) \in U$. Then, for any $\epsilon>0$ sucb that $U \cap(\tau+\epsilon, T)$ $\equiv U_{\epsilon} \neq \varnothing, \inf \left\{G_{U}(z ; \zeta) ; z \in U_{\epsilon}\right\}=0$.

Proof. Assume for contradiction that $\inf \left\{G_{U}(z ; \zeta) ; z \in U_{\epsilon}\right\}=c>0$. Let $\Sigma_{1}, \Sigma_{2} \subset E^{n}$ be balls such that $\bar{U}_{\epsilon} \subset \Sigma_{1} \times[\tau+\epsilon, T]$ and $\bar{U} \cap[0, \tau+\epsilon]$ $\subset \Sigma_{2} \times[0, \tau+\epsilon]$. Let $b_{\zeta}$ be the solution of the boundary value problem

$$
\begin{aligned}
L u=0 & \text { in } \Sigma_{1} \times(\tau+\epsilon, T), \\
u(x, t)=c\left(\frac{t-\tau-\epsilon}{T-\tau-\epsilon}\right) & \text { on } \partial \Sigma_{1} \times[\tau+\epsilon, T], \\
u(x, \tau+\epsilon)=0 & \text { on } \Sigma_{1} .
\end{aligned}
$$

Then by the minimum principle, Theorem $4,0 \leq b_{\zeta} \leq c$ on $\Sigma_{1} \times(\tau+\epsilon, T)$. Moreover, it follows from Trudinger's results [6] that $b_{\zeta}>0$ on $\Sigma_{1} \times$ $(\tau+\epsilon, T)$ and $b_{\zeta} \in C\left(\bar{\Sigma}_{1} \times[\tau+\epsilon, T]\right)$. Extend the domain of $b_{\zeta}$ by setting $b_{\zeta} \equiv 0$ in $\Sigma_{2} \times[0, \tau+\epsilon]$. Then, by the extension principle [1], $b_{\zeta}$ satisfies $L u=0$ weakly in

$$
\left\{\Sigma_{1} \times(\tau+\epsilon, T)\right\} \cup\left\{\Sigma_{2} \times(0, \tau+\epsilon]\right\} \supset U
$$


Define

$$
F(z ; \zeta) \equiv G_{U}(z ; \zeta)-h_{\zeta}(z) \text { on } U
$$

Then $F$ satisfies the following properties:

(1) $F(z ; \zeta)=\Gamma(z ; \zeta)+H(z ; \zeta)-b_{\zeta}(z)$ where $H(\cdot ; \zeta)-b_{\zeta}$ satisfies $L u=0$ in $U$.

(2) On $U_{\epsilon}, b_{\zeta} \leq c$ and $G_{U} \geq c$ imply $F(\cdot ; \zeta) \geq 0$ and on $U-U_{\epsilon}, b_{\zeta} \equiv$ 0 implies $F(\cdot ; \zeta)=G_{U}(\cdot ; \zeta) \geq 0$. Thus $F(\cdot ; \zeta) \geq 0$ on $U$.

(3) On $U_{\epsilon}, b_{\zeta}>0$ implies $F(\cdot ; \zeta)<G_{U}(\cdot ; \zeta)$ and this contradicts the minimality condition on $G_{U}$.

Therefore, $\inf \left\{G_{U}(\bullet ; \zeta) ; z \in U_{\epsilon}\right\}=0$.

Lemma 6. If the open set $U$ bas a Green function $G_{U}$ and if $R$ is a standard rectangle with $\zeta \in R$ and $\bar{R} \subset U$, then $G_{U}(\because \zeta)$ is bounded on $U-R$.

Proof. The proof of this lemma is quite simple if it is noted that property (3) of $G_{U}$ and Theorem 7 of [1] imply that

$$
0 \leq G_{U}(x, t ; \xi, \tau) \leq \Gamma(x, t ; \xi, \tau) \leq C g_{\beta}(x-\xi, t-\tau)
$$

where $g_{\beta}(x, t)$ is the fundamental solution of $u_{t}-\beta \Delta u=0$ in $E^{n} \times(0, T)$.

Definition 14. If $U$ is a domain in $E^{n} \times(0, T)$ and $\zeta \in U$, define

$$
B_{\zeta}=\left\{v_{\zeta} ; v_{\zeta} \geq 0, v_{\zeta}=\Gamma(\cdot ; \zeta)+w_{\zeta}, w_{\zeta} \in \mathcal{S}_{U}^{\prime}\right\}
$$

Note that, in contrast to the case where $L$ is replaced with the Laplace operator $\Delta, \mathscr{P}_{\zeta} \neq \varnothing$ for all $\zeta \in U$. The proof of the following lemma is almost identical to the proof for the Laplace operator found in [6].

Lemma 7. $B_{\zeta}$ is a saturated family of functions on $U-\{\zeta\}$.

An obvious consequence of the following theorem is that any open set $U$ in $E^{n} \times(0, T)$ has a Green function.

Theorem 19. If $B_{\zeta} \neq \varnothing$ for each $\zeta \in U$, then $U$ bas a Green function $G_{U}(\cdot ; \zeta)=\inf \left\{v_{\zeta} ; v_{\zeta} \in \mathscr{P}_{\zeta}\right\}$.

Proof. Let $\zeta \in U$ be fixed and let $R$ be a standard rectangle with $\zeta \in R, \bar{R} \subset U$. For any $u \in \mathcal{S}_{U}^{\prime}$ define $u^{*}$ by

$$
u^{*}(z)= \begin{cases}L(u ; z, R), & z \in R, \\ u(z), & z \in U-R .\end{cases}
$$


Then, by Theorem $10^{\prime}, u^{*} \in \mathcal{S}_{U}^{\prime}$. Hence, if $v_{\zeta} \equiv \Gamma(\cdot ; \zeta)+b_{\zeta} \in B_{\zeta}$, then $\bar{v}_{\zeta} \equiv \Gamma(\cdot ; \zeta)+b_{\zeta}^{*} \in \Re_{\zeta \cdot}$ Therefore, since $u^{*} \leq u$ on $U$,

$$
G_{U}(\cdot ; \zeta)=\inf \left\{\bar{v}_{\zeta} ; v_{\zeta} \in B_{\zeta}\right\}=\Gamma(\cdot ; \zeta)+\inf \left\{h_{\zeta}^{*} ; h_{\zeta} \geq-\Gamma(\cdot ; \zeta), h_{\zeta} \in \mathcal{S}_{U}^{\prime}\right\} .
$$

It follows from Theorem 13 and the fact that $b_{\zeta} \geq-\Gamma(\cdot ; \zeta)$ that

$$
H(\cdot ; \zeta) \equiv \inf \left\{h_{\zeta}^{*} ; h_{\zeta} \geq-\Gamma(\cdot ; \zeta), h_{\zeta} \in \mathcal{S}_{U}^{\prime}\right\}
$$

is parabolic on $U-\{\zeta\}$. Moreover, since each $b_{\zeta}^{*}$ is parabolic on $R$, a neighborhood of $\zeta$, and since $b_{\zeta}^{*} \geq-\Gamma(\cdot ; \zeta)$ on $\partial_{\rho} R$, Lemma 3 says that $H(\cdot ; \zeta)$ is parabolic on $R$. Therefore, $H(\cdot ; \zeta)$ is parabolic on $U$ and $G_{U}(\bullet \zeta)=\Gamma(\bullet ; \zeta)+H(\cdot ; \zeta)$.

Now each $v_{\zeta} \in \mathscr{P}_{\zeta}$ satisfies $v_{\zeta} \geq 0$ and, hence, $G_{U}(\cdot ; \zeta) \geq 0$. Suppose $v=\Gamma(\cdot ; \zeta)+b_{\zeta}$ where $b_{\zeta} \in \mathcal{S}_{U}^{\prime}$ and $v \geq 0$. Then $v \in \mathscr{P}_{\zeta}$ and, by the definition of $G_{U}, v \geq G_{U}(\cdot ; \zeta)$.

The proofs of the following three theorems are identical with those for the corresponding results of the Laplace operator and are not given here.

Theorem 20. If $U_{1} \subset U_{2}$ are domains in $E^{n} \times(0, T)$ with Green functions $G_{1}$ and $G_{2}$, respectively, then $G_{1} \leq G_{2}$ on $U_{1} \times U_{1}$.

Theorem 21. If the domain $U$ bas a Green function $G$, then the greatest parabolic minorant of $G(\cdot ; \zeta), \zeta \in U$, is the zero function.

Theorem 22. Let $\left\{U_{j}\right\}$ be a monotone increasing sequence of domains in $E^{n} \times(0, T), U=\bigcup_{j=1}^{\infty} U_{j}$, and assume $U_{j}$ bas a Green function $G_{j}$. If $U$ bas a Green function $G_{U}$, then $G_{j} \uparrow G_{U}$ on $U \times U$.

It follows from Theorem 22 that the equivalence of $G_{U}$ and $\gamma$ when $U=Q$ will follow if it can be shown for sets $Q$ where $\partial \Omega$ is smooth.

Theorem 23. Let $\Omega \subset E^{n}$ be a domain with $\partial \Omega$ smooth. For any $z$, $\zeta \in Q$ with $z \neq \zeta, \gamma(z ; \zeta)=G_{Q}(z ; \zeta)$

Proof. Let $\left\{L^{m}\right\}$ be a sequence of smooth operators which have been obtained from $L$ by mollification. Let $\gamma^{m}$ and $\Gamma^{m}$ be the Green and fundamental solutions of $L^{m} u=0$ in $Q$ and $E^{n} \times(0, T)$, respectively. Friedman [4] has shown that for each $\zeta \in Q$ there is a parabolic function $V^{m}$ on $Q$ such that $\gamma^{m}(\bullet ; \zeta)=\Gamma^{m}(\bullet ; \zeta)+V^{m}(\bullet ; \zeta)$. It follows from a theorem of Aronson's that there is a subsequence of $\{m\}$, also denoted by $\{m\}$, such that $\gamma^{m}(\bullet ; \zeta) \rightarrow \gamma(\bullet \zeta \zeta), \Gamma^{m}(\bullet \zeta \zeta) \rightarrow \Gamma(\bullet ; \zeta)$ uniformly on all compact subsets of $Q-\{\zeta\}$. Therefore, $V^{m}(\cdot ; \zeta) \rightarrow V(\cdot ; \zeta)=\gamma(\cdot ; \zeta)-\Gamma(\cdot ; \zeta)$ uniformly on all compact subsets of $Q-\{\zeta\}$. 
Now each $V^{m}(\because \zeta \zeta)$ is parabolic in $Q$ and $\zeta \in Q$. Thus, for any standard rectangle $R$ with $\zeta \in R$ and $\bar{R} \subset Q,\left\{V^{m}(\because \zeta \zeta)\right\}$ is an equicontinuous family on $\bar{R}$ and, by Arzela's theorem, there is a uniformly convergent subsequence. Passing through this subsequence it follows from Lemma 2 that $V(\because \zeta \zeta)$ is parabolic on $R$. Hence, $V(\cdot ; \zeta)$ is parabolic on $Q$. That is, $\gamma(\cdot \zeta \zeta)=\Gamma(\cdot ; \zeta)+V(\cdot ; \zeta)$ and property (1) of Definition 13 is satisfied.

It follows from [1] that $\gamma \geq 0$ and property (2) is satisfied. Next suppose $V(\bullet ; \zeta)=\Gamma(\bullet ; \zeta)+W(\bullet ; \zeta)$ where $W(\bullet \zeta \zeta) \in \mathcal{S}_{Q}^{\prime}$ for each $\zeta \in Q$. Set $W(\bullet ; \zeta)=v(\cdot ; \zeta)-\gamma(\cdot ; \zeta)$. Then $W=w-V \in \mathcal{S}_{Q}^{\prime}$ since $V(\bullet ; \zeta)$ is parabolic. Since $\partial \Omega$ is smooth, it follows from [1] that $\gamma(\bullet \zeta \zeta)=0$ on $\partial \Omega \times$ $(0, T)$. Moreover $\gamma(z ; \zeta)=0$ for $z \prec \zeta$ and $\gamma(\because \zeta) \in C(\Omega \times[0, T)-\{\zeta\})$. Therefore, $\lim _{z \rightarrow \partial_{\rho} Q} W(\because \zeta) \geq 0$.

It follows from Theorem 4 that $W(\cdot ; \zeta) \geq 0$ on $Q$. Hence property (3) is satisfied. Since $G_{Q}$ is unique and since $\zeta \in Q$ was arbitrary, $\gamma=G_{Q}$ on $Q \times Q$.

\section{REFERENCES}

1. D. G. Aronson, Non-negative solutions of linear parabolic equations, Ann. Scuola Norm. Sup. Pisa 22 (1968), 607-694.

2. Neil Eklund, Existence and representation of solutions of parabolic equations, Proc. Amer. Math. Soc. 47 (1975), 137-142.

3. - Generalized supersolutions of parabolic equations (submitted).

4. - Convergent nets of parabolic and generalized superparabolic functions, Proc. Amer. Math. Soc. 50 (1975), 237-243.

5. L. L. Helms, Introduction to potential theory, Pure and Appl. Math., vol. 22, Interscience, New York, 1969. MR 41 \# 5638.

6. Neil Trudinger, Pointwise estimates and quasilinear parabolic equations, Comm. Pure Appl. Math. 21 (1968), 205-226. MR 37 \#1758.

DEP ARTMENT OF MATHEMATICS, VANDERBILT UNIVERSITY, NASHVILLE, TENNESSEE 37235

Current address: Department of Mathematics, Centre College, Danville, Kentucky 40422 